01

\title{
Трехуровневое приближение при расчете параметров оптически детектируемого магнитного резонанса в условиях сильной лазерной накачки
}

\author{
(C) А.К. Вершовский, М.В. Петренко \\ Физико-технический институт им. А.Ф. Иофффе РАН, \\ Санкт-Петербург, Россия \\ e-mail: antver@mail.ioffe.ru
}

Поступила в редакцию 05.12.2020 г.

В окончательной редакции 05.12.2020 г.

Принята к публикации 17.12.2020 г.

Предложен алгоритм приближенного решения сугубо нелинейной задачи о параметрах оптически детектируемого магнитного резонанса в основном состоянии щелочных атомов в оптически плотной среде в условиях сильной узкополосной оптической накачки, вызывающей, во-первых, просветление атомарной среды, во-вторых - частичное подавление спин-обменного уширения. Непосредственное решение уравнения Лиувилля в этих условиях осложняется тем, что время релаксации каждого из уровней сверхтонкой и зеемановской структур основного состояния определяется населенностями остальных уровней, что приводит к необходимости решения самосогласованной задачи в многоуровневой системе, и, как правило, требует использования суперкомпьютеров. В данной работе предложены приближения, позволяющие существенно упростить и на порядки ускорить расчет, и проведено сравнение результатов с данными эксперимента на примере двухлучевой $M_{X}$-схемы магнитометрического датчика.

Ключевые слова: оптически детектируемый магнитный резонанс, квантовый магнитометр, трехуровневое приближение, уравнения Блоха, балансные уравнения.

DOI: $10.21883 / \mathrm{OS} .2021 .04 .50775 .300-20$

\section{Введение}

Последние годы характеризуются стремительным увеличением интереса к компактным квантовым датчикам с оптической накачкой $(\mathrm{OH})$ и оптическим детектированием (ОД) магнитного резонанса (МР). К этим устройствам относятся магнитометры, гироскопы и, в какой-то степени, стандарты частоты. Все эти устройства характеризуются непревзойденным сочетанием чувствительности, точности, и быстродействия [1]. Практический интерес к магнитометрам последнего поколения не в последнюю очередь обусловлен перспективностью их использования в магнитоэнцефалографических (МЭГ) комплексах [2] и системах магнитнорезонансной томографии (МРТ) сверхслабого поля [3]. Одновременно происходит бурное развитие гироскопов, использующих в одной схеме как ядерный магнитный резонанс, так и оптическое детектирование магнитного резонанса (ОДМР). Все это стало возможным благодаря появлению компактных одномодовых лазерных систем и развитию методов лазерной накачки МР.

Расчет параметров МР в таких датчиков осложнен, во-первых, тем, что они используют оптически плотную среду, что, как правило, требует применения численных приближенных методов [4]. Далее, достижение оптимальной плотности среды в компактных $(<1 \mathrm{~cm})$ газовых ячейках, содержащих насыщенный пар щелочного металла, подразумевает высокие концентрации паров, следовательно - работу при температурах, при которых безусловно доминирующим фактором, определяющим ширину линии, является спин-обменное уширение. Как показал У. Хаппер с коллегами [5,6], спин-обменное уширение отдельных подуровней зеемановской структуры зависит не только от температуры, но и от распределения населенностей этих подуровней - чем больше атомов сконцентрировано на данном подуровне, тем меньше остается атомов, столкновение с которыми может привести к перераспределению значений углового момента, и тем меньше спин-обменное уширение этого уровня. Состояние, в котором подавляющее большинство атомов сконцентрировано на одном подуровне и спин-обменное уширение практически подавлено, У. Хаппер назвал „вытянутым“ (stretched) состоянием [7]. Как показано в [7], достижение „вытянутого“ состояния (и соответственно реализация предельных практически не ограниченных спин-обменом чувствительностей) возможно при сильной лазерной накачке в ненулевых магнитных полях. Использование режима „вытянутого“ состояния позволяет датчикам ненулевого поля конкурировать с датчиками, работающими в режиме SERF (spin-exchange relaxation free, полное подавление спин-обменного уширения линии МР в нулевом магнитном поле) $[8,9]$, в том числе и в системах МЭГ $[6,10]$.

Реализовать „вытянутое“ состояние проще всего применением сильной лазерной накачки циркулярно по- 
ляризованным $(\sigma)$ светом $D_{1}$-линии, настроенным в резонанс с переходом $F=I-1 / 2 \leftrightarrow F^{\prime}=I \pm 1 / 2$ из основного состояния $S_{1 / 2}$, где $I-$ момент ядра (для Cs $I=7 / 2), F$ и $F^{\prime}$ - полные моменты основного и возбужденного состояния соответственно $[5,11]$. Наиболее перспективной является схема детектирования МР на переходе $F=I+1 / 2, m_{F}=F \leftrightarrow F-1$ из основного состояния на ларморовой частоте (так называемая $M_{X}$-схема) по повороту поляризации нерезонансного линейно поляризованного поперечного пробного луча, отстроенного от соответствующей оптической линии поглощения вниз по частоте на несколько ширин оптической линии [12]. Но возможны и другие варианты детектирование на частоте модуляции радиочастотного поля $\left(M_{Z}\right.$-схема), детектирование по поглощению луча накачки (однолучевая схема) и т.д.

Мы в данной работе будем рассматривать „классическую“ двухлучевую $M_{X}$-схему, в которой луч накачки направлен вдоль вектора внешнего магнитного поля $\mathbf{B}_{\mathbf{0}}$, а МР возбуждается резонансным радиочастотным полем (радиополем), и ее модификацию - схему БеллаБлюма [13], резонанс в которой возбуждается посредством модуляции параметров перпендикулярной к $\mathbf{B}_{0}$ оптической накачки [14]. Как будет показано ниже, обе эти схемы хорошо описываются в рамках предложенных нами приближений, хотя и требуют введения разных коэффициентов, описывающих световое уширение и подавление спин-обменного уширения.

\section{Теория}

Рассмотрим схему подуровней основного состояния щелочного металла. Каждый сверхтонкий уровень $F=I \pm 1 / 2$ в магнитном поле расщепляется на $2 F+1$ подуровней с проекциями момента $m_{F}=-F, \ldots, F$. Общее количество этих подуровней равно $N=2(2 I+1)$ (в случае атома Cs $N=16)$. В равновесном состоянии подуровни заселены одинаково, поскольку разность их энергий пренебрежимо мала по сравнению с $k T$. Зная скорости релаксации и откачки с этих подуровней, значения населенностей можно получить, решив систему из $N$ балансных уравнений. В простейшем случае, предполагая, что разность населенностей в системе достигается только за счет разности скоростей откачки, а вероятности распада из возбужденного состояния во все уровни основного состояния равны (случай полного перемешивания в возбужденном состоянии), получаем систему линейных балансных уравнений вида

$$
\frac{d n_{i}}{d t}=-\Gamma_{i} n_{i}+\frac{1}{2(2 I+1)} \sum_{j} \Gamma_{j} n_{j},
$$

где $i, j=1 \ldots N, n_{i}$ - населенности уровней, $\Gamma_{0}-$ общая темновая скорость релаксации, $\Gamma_{i}=\Gamma_{0}+R_{i}-$ скорости их релаксации, включающие в себя скорости оптической откачки $R_{i}$. В стационарном случае система

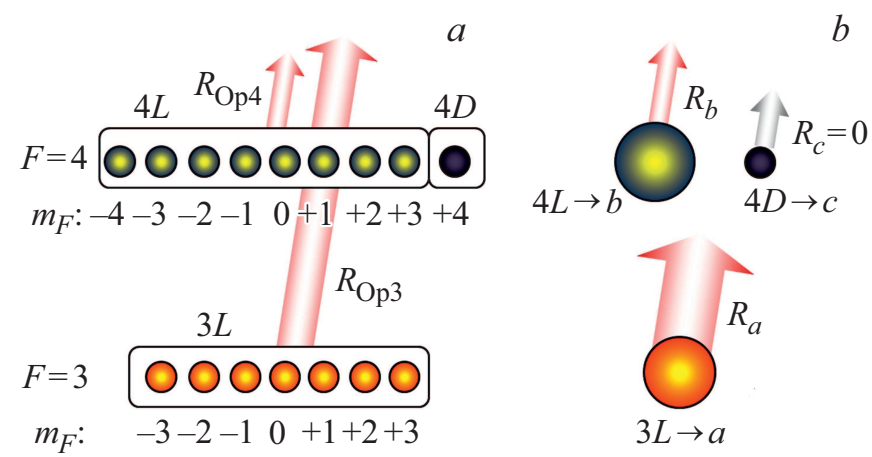

Рис. 1. (a) Схема магнитных подуровней основного состояния Cs (обозначения в тексте), (b) трехуровневое приближение для основного состояния Cs.

решается приравниванием левой части нулю с учетом условия нормировки $\sum_{i} n_{i}=1$.

Если нас интересуют не только населенности, но и когерентности, то мы должны решать систему из $\left(N^{2}+N\right) / 2$ независимых уравнений Лиувилля для матрицы плотности. Такая задача неоднократно решалась для случая как оптически тонкого, так и толстого слоя, как с учетом движения атомов, так и без него [4]. Однако учет „вытянутого“ состояния катастрофически усложняет эту задачу, поскольку $\Gamma_{i}$ оказываются зависимыми от вcex $n_{j}$.

Поэтому для того, чтобы довести задачу до численного решения, ее необходимо упростить, насколько это возможно. Рассмотрим случай накачки атома Cs циркулярно поляризованным $(\sigma+)$ светом $D_{1}$-линии в ячейке с буферным газом. Будем считать, что давление газа достаточно велико для того, чтобы, во-первых, эффективно (хотя бы на уровне единиц процентов) перекрывались оптические линии поглощения с двух сверхтонких уровней возбужденного состояния, и, вовторых, происходило бы эффективное перемешивание электронных состояний в возбужденном состоянии. Скорости оптической откачки с уровней $F=3,4$ обозначим соответственно $R_{\mathrm{Op} 3}$ и $R_{\mathrm{Op} 4}\left(R_{\mathrm{Op} 3} \gg R_{\mathrm{Op} 4}\right)$. Будем считать, что в отсутствие оптической накачки доминирующий (хотя и не единственный) вклад в скорость релаксации дает спин-обмен.

Сгруппируем 16 подуровней основного состояния $\mathrm{Cs}$ в три группы (рис. $1, a)$ :

1. Группа $3 L$ : взаимодействующие со светом подуровни на $F=3\left(m_{F}=-3 \ldots+3\right)$;

2. Группа $4 L$ : взаимодействующие со светом подуровни на $F=4\left(m_{F}=-4 \ldots+3\right)$;

3. Группа $4 D$ : не взаимодействующий со светом (,темный“) подуровень на $F=4\left(m_{F}=+4\right)$.

Будем рассматривать каждую группу, как отдельный уровень $a, b, c$ (рис. $1, b)$. Такое приближение оправдано не только сходством условий, в которых находятся подуровни внутри каждой группы, но и тем, что в дальнейшем нас будет интересовать только резонанс, 
соответствующий переходу с подуровня $F=4, m_{F}=+4$ (группа $4 D$ или уровень $c$ - так называемый „карман“, который не взаимодействует со светом, и в котором в результате сильной оптической накачки собирается большая часть атомов) на соседний уровень (группа $4 L$ ), и не будут интересовать переходы внутри групп. Различиями в скоростях накачки внутри каждой группы пренебрегаем.

Получаем трехуровневую систему - три уровня с весами $C_{i}\left(i=a, b, c\right.$, для Cs при накачке $D_{1}$-линией $\left.C_{a}=7, C_{b}=8, C_{c}=1\right)$. Все уровни характеризуются общей темновой скоростью релаксации $\Gamma_{0}$, но каждый уровень характеризуется своей скоростью откачки $R_{i}$, причем $R_{c}=0$. Обозначим суммарные населенности каждой группы $N_{i}$, а $n_{i}=N_{i} / C_{i}-$ среднюю населенность подуровня в группе. Введем безразмерные скорости накачки $r_{i}=R_{i} / \Gamma_{0}$ и релаксации в присутствии накачки $g_{i}=\left(\Gamma_{0}+R_{i}\right) / \Gamma_{0}=r_{i}+1$, при этом $r_{c}=0$ и $g_{c}=1$.

Учтем два эффекта, оказывающих определяющее влияние на распределение населенностей в системе: 1) частичный перенос момента с уровней $a$ и $b$ на уровень $c$ при оптической накачке, характеризующийся коэффициентом переноса $\alpha$, причем $0 \leq \alpha \leq 1$; физически перенос момента объясняется сохранением ядерной компоненты момента в возбужденном состоянии [11]; 2) снижение скорости спин-обменной релаксации уровня $c$ в „вытянутом“ состоянии:

$$
g_{c}=\frac{1}{1+k_{l n}},
$$

где $k_{l n} \geq 0-$ коэффициент сужения линии МР. С учетом того, что $C_{c}=1$, получаем систему балансных уравнений:

$$
\begin{aligned}
& \frac{1}{\Gamma_{0}} \frac{d n_{a}}{d t}=-g_{a} n_{a}+\frac{1}{M}\left[g_{a} C_{a} n_{a}+g_{b} C_{b} n_{b}+g_{c} n_{c}\right], \\
& \frac{1}{\Gamma_{0}} \frac{d n_{b}}{d t}=-g_{b} n_{b}+\frac{1}{M}\left[\left(g_{a}-\alpha r_{a}\right) C_{a} n_{a}\right. \\
& +\left(g_{b}-\alpha\left(g_{b}-1\right)\right) C_{b} n_{b} \\
& \left.+\left(g_{c}-\alpha\left(g_{c}-1\right)\right) n_{c}\right], \\
& \frac{1}{\Gamma_{0}} \frac{d n_{c}}{d t}=-g_{c} n_{c}+\frac{1}{M}\left[\left(g_{a}+C_{b} \alpha\left(g_{a}-1\right)\right) C_{a} n_{a}\right. \\
& \left.+\left(g_{b}+C_{b} \alpha\left(g_{b}-1\right)\right) C_{b} n_{b}+\left(g_{c}+C_{b} \alpha\left(g_{c}-1\right)\right) n_{c}\right] .
\end{aligned}
$$

Приравнивая левые части уравнений нулю (стационарное решение) и учитывая условия нормировки, получаем решение системы уравнений (3) в стационарном состоянии:

$$
\begin{gathered}
n_{a}=\frac{1}{R}\left[2+k_{l n} \alpha+r_{b}\left(2+\left(1+k_{l n}\right) \alpha\right)\right], \\
n_{b}=\frac{1}{R}\left[2+\frac{9}{8} k_{l n} \alpha+r_{a}\left(2+\frac{1}{8}\left(9 k_{l n}-7\right) \alpha\right)\right],
\end{gathered}
$$

$$
\begin{aligned}
& n_{c}=\frac{1}{R}\left[( 1 + k _ { l n } ) \left(2+r_{b}(2+9 \alpha)\right.\right. \\
& \left.\left.+r_{a}\left(2+7 \alpha+2 r_{b}(1+8 \alpha)\right)\right)\right], \\
& R=2\left[k_{l n}\left(1+r_{a}\right)\left(1+r_{b}\right)(1+8 \alpha)\right. \\
& +8\left(2+r_{a}+\alpha r_{b}\right) \\
& \left.+\left(9(1+8 \alpha) r_{b}\right) r_{a}\right] .
\end{aligned}
$$

Предположим, что скорость спин-обменной релаксации атомов, находящихся в группе $c$, пропорциональна суммарной концентрации атомов, находящихся в группах $a$ и $b$, (которая, в свою очередь, равна $\left.n_{a}+n_{b}=1-n_{c}\right)$. Тогда

$$
k_{l n}=k_{n}\left(\frac{N-1}{N} \frac{1}{1-n_{c}}-1\right)
$$

где $k_{n}-$ корректирующий коэффициент (предположительно $\left.k_{n} \approx 1\right)$, а множитель $(N-1) / N$ введен для нормировки: в равновесном состоянии сужение линии отсутствует, и $k_{l n}=0$. Выражение (5) можно подставить в (2) и затем в (3) или сразу в (4), и получить систему нелинейных уравнений, но проще решать (4) последовательными итерациями.

Как следует из рис. 2 , для практических целей достаточно второй итерации. Видно, что результат существенно зависит от коэффициента переноса $\alpha$ : наличие переноса позволяет достичь высоких степеней поляризации среды при меньших скоростях оптической накачки.

При расчетах сечения поглощения лазерного излучения и темновой ширины линии были использованы выражения из [7,15-17]. Коэффициент поглощения излучения накачки в ячейке рассчитывался, исходя из распределения населенностей, установившегося под воздействием света накачки. Эту задачу мы также решали итерациями, ограничиваясь при этом подсчетом средних по ячейке величин. Так, среднюю по ячейке относительную интенсивность накачки $I_{\text {pMean }}$ при экспоненциальном поглощении можно получить интегрированием по длине ячейки:

$$
I_{\mathrm{pMean}}=\frac{1}{k_{\nu 3}+k_{v 4}}\left(1-I_{p 0} e^{-k_{v i} L}\right),
$$

где $k_{\nu 3}, k_{\nu 4}$ - значения коэффициентов поглощения излучения уровнями $F=3,4$. При этом на каждой итерации входящие в выражения для $k_{v 3}, k_{v 4}$ концентрации атомов на соответствующих уровнях вычисляются согласно формулам (4), после чего полученное значение средней интенсивности используется для вычисления скоростей накачки $r_{a}, r_{b}$. Двух таких итераций оказывается достаточно, чтобы адекватно описать просветление среды. 


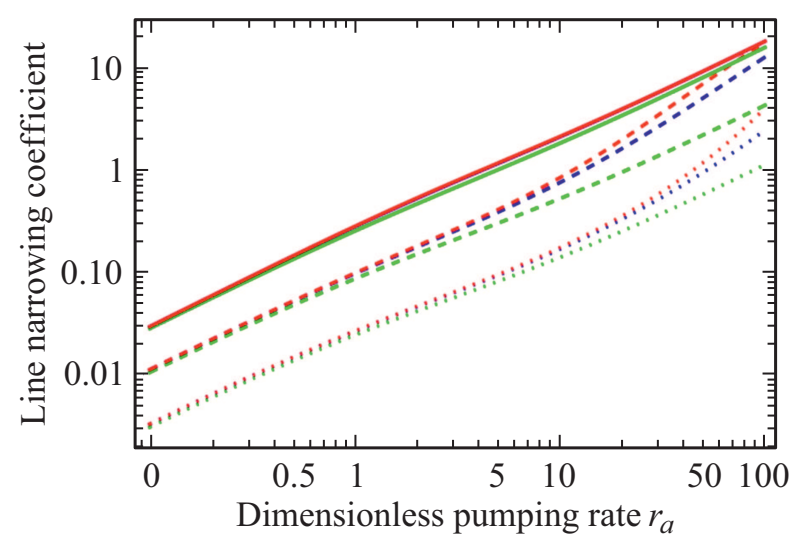

$b$

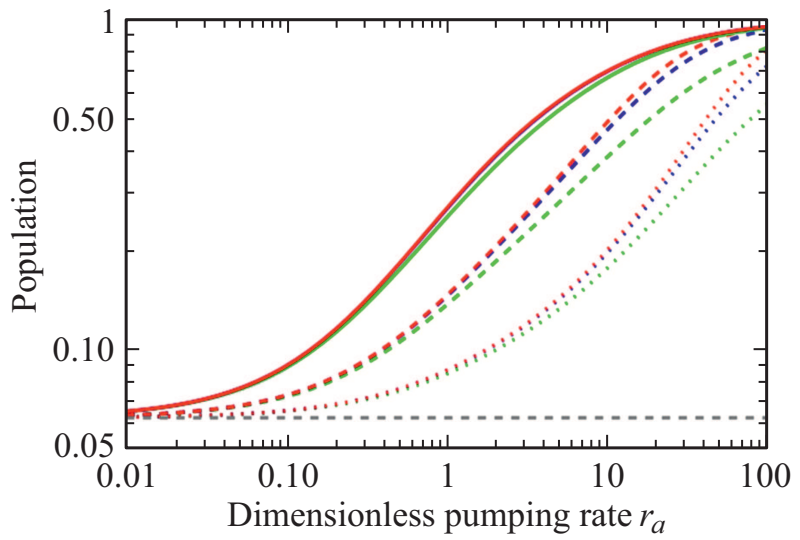

Рис. 2. Зависимость вычисленного по формулам (4),(5) коэффициента сужения линии $k_{\ln }$ и населенности $n_{c}$ уровня $F=4$, $m_{F}=+4$ от скорости накачки $r_{a}$ при $r_{b} / r_{a}=0.1$. Коэффициент переноса $\alpha=0$ (пунктирная кривая), 0.3 (штриховая кривая), 1 (сплошная кривая); зеленые кривые - 1-я итерация, синие кривые - 2-я итерация, красные кривые - 3-я итерация. $(a)$ Коэффициент сужения линии, $(b)$ населенность $n_{c}$. Серая штриховая линия - равновесная заселенность.

$a$

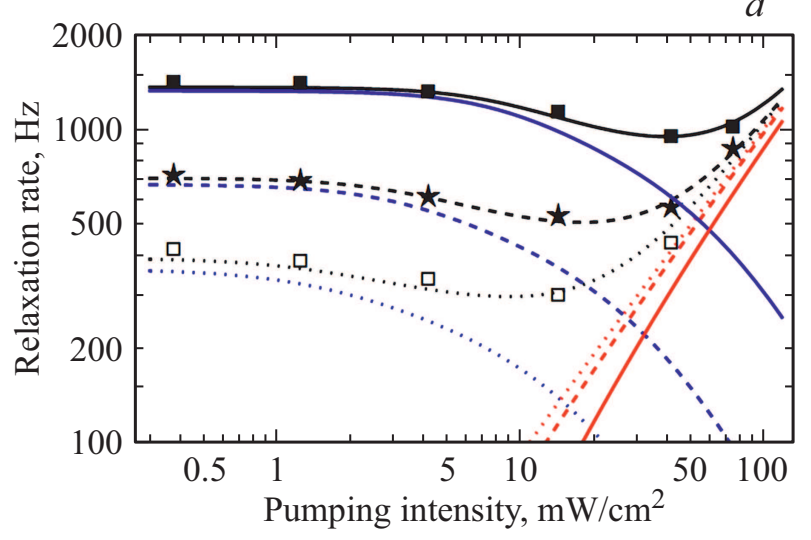

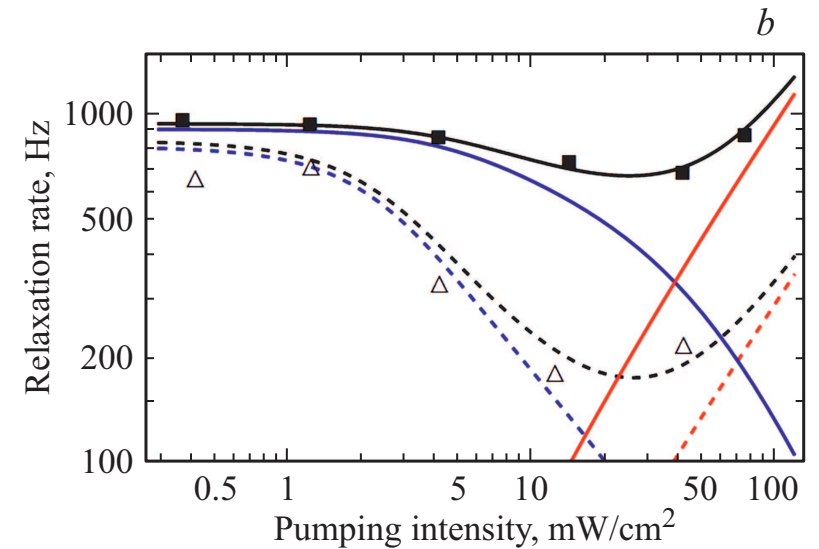

Рис. 3. Полуширина резонанса в зависимости от интенсивности накачки $I_{p}$, интенсивность пробного луча $I_{d}=4.5 \mathrm{~mW} / \mathrm{cm}^{2}$, символы - эксперимент, сплошные кривые - расчет. Синиие кривые - спин-обменное уширение, красные кривые уширение светом накачки, черные кривые - полная ширина. (a) Схема Белла-Блюма при $T=95^{\circ} \mathrm{C}$ (пунктирные кривые), $105^{\circ} \mathrm{C}$ (штриховые кривые), $115^{\circ} \mathrm{C}$ (сплошная кривые). (b) „Классическая“ схема (штриховые кривые) и схема Белла-Блюма (сплошные кривые) при $110^{\circ} \mathrm{C}$.

\section{Сравнение с экспериментом}

Экспериментальная установка, на которой производились измерения, описана в $[10,18]$. Магнитный резонанс возбуждался в кубической ячейке размером $8 \times 8 \times 8 \mathrm{~mm}$, содержащей насыщенные пары Cs и азот под давлением $\sim 100$ Torr. Магнитный резонанс исследовался как в „классической“ двухлучевой конфигурации, так и в схеме Белла-Блюма при синусоидальной поляризации луча накачки. Максимальная мощность излучения накачки на входе в ячейку составляла $\sim 15 \mathrm{~mW}$ на длине волны $795 \mathrm{~nm}$ ( $D_{1}$-линия $\left.\mathrm{Cs}\right)$.

Результаты сопоставления теории с экспериментом приведены на рис. 3. При расчете использовались подгоночные коэффициенты, а именно - введенный ранее коэффициент, корректирующий световое сужение линии $k_{n}$, и коэффициент $k_{b}$, аналогичным образом корректирующий световое уширение линии. Как оказалось, значения этих коэффициентов существенно отличаются для „классической“ схемы и для схемы Белла-Блюма: в схеме Белла-Блюма коэффициент светового уширения линии оказался в 3.3 раза больше, а коэффициент светового сужения линии —в 7.2 раза меньше; это иллюстрируется рис. $3, b$. Поскольку степень светового сужения линии определяется степенью „вытянутости“ состояния, это означает, что в схеме Белла-Блюма при той же степени поляризации среды нам не удалось достичь той же степени „вытянутости“ состояния. Это можно объяснить меньшей степенью сфазированности прецессии находящихся в одном и том же состоянии атомных моментов при возбуждении резонанса модуляцией параметров оптической накачки. Изучению этого 
эффекта посвящены наши дальнейшие исследования. Поскольку представленная в данной работе модель позволяет вычислять времена релаксации уровней, но не когерентностей, соответствующие поправки приходится вводить феноменологически. Тем не менее, из рис. 3 следует, что модель позволяет получить адекватное описание параметров МР в широком диапазоне условий, в том числе и при температурах, при которых оптическая толщина ячейки оказывается много большей единицы.

\section{Заключение}

Нами предложен и проверен сопоставлением с экспериментом упрощенный метод расчета параметров МР в схеме квантовых оптических магнитных датчиков ненулевого поля. Метод позволяет простыми средствами рассчитывать ширину МР в условиях оптически толстой ячейки и нелинейного подавления спин-обменного уширения сильным лазерным излучением накачки.

\section{Конфликт интересов}

Авторы заявляют, что у них нет конфликта интересов. Вклад авторов: эксперимент - М.В. Петренко, А.К. Вершовский; теория - А.К. Вершовский.

\section{Финансирование работы}

Работа выполнена при финансовой поддержке РФФИ в рамках научного проекта№ 19-29-10004.

\section{Список литературы}

[1] Budker D., Romalis M. // Nat. Phys. 2007. V. 3. P. 227. doi $10.1038 /$ nphys 566 .

[2] Hämäläinen M., Hari R., Ilmoniemi R.J., Knuutila J., Lounasmaa O.V. // Rev. Mod. Phys. 1993. V. 65. N 2. P. 413. doi 10.1103/RevModPhys.65.413

[3] Coffey A.M., Truong M.L., Chekmenev E.Y. // J. Magn. Res. 2013. V. 237. P. 169. doi 10.1016/j.jmr.2013.10.013

[4] Баранцев К.А. и др. // ЖЭТФ. 2021. Т. 159. № 1.

[5] Scholtes T., Schultze V., IJsselsteijn R., Woetzel S., Meyer H.-G. // Phys. Rev. A. 2011. V. 84. N 4. P. 043416. doi 10.1103/PhysRevA.84.043416

[6] Schultze V., Schillig B., IJsselsteijn R., Scholtes T., Woetzel S., Stolz R. // Sensors. 2017. V. 17. N 3. P. 561. doi 10.3390/s17030561

[7] Bhaskar N.D., Camparo J., Happer W., Sharma A. // Phys. Rev. A. 1981. V. 23. N 6. P. 3048. doi 10.1103/PhysRevA.23.3048

[8] Kominis I.K., Kornack T.W., Allred J.C., Romalis M.V. // Nature. 2003. V. 422. N 6932. P. 596. doi 10.1038/nature01484

[9] Dang H.B., Maloof A.C., Romalis M.V. // Appl. Phys. Lett. 2010. V. 97. N 15. P. 151110. doi 10.1063/1.3491215
[10] Вершовский А.К., Пазгалев А.С., Петренко М.В. // Письма в ЖТФ. 2020. Т. 46. № 17. С. 43. doi 10.21883/PJTF.2020.17.49894.18340; Vershovskii A.K., Pazgalev A.S., Petrenko M.V. // Tech. Phys. Lett. 2020. V. 46. N 9. P. 877. doi 10.1134/S1063785020090126

[11] Попов Е.Н. и др. // Письма в ЖЭТФ. 2018. Т. 108. № 8. C. 543. doi 10.1134/S0370274X18200043; Popov E.N. et al. // JETP Lett. 2018. V. 108. N 8. P. 513. doi 10.1134/S0021364018200122

[12] Budker D., Kimball D.F., Rochester S.M., Yashchuk V.V., Zolotorev M. // Phys. Rev. A. 2000. V. 62. N 4. P. 043403. doi 10.1103/PhysRevA.62.043403

[13] Bell W.E., Bloom A.L. // Phys. Rev. Lett. 1961. V. 6. N 6. P. 280. doi 10.1103/PhysRevLett.6.280

[14] Fescenko I., Knowles P., Weis A., Breschi E. // Opt. Express. 2013. V. 21. N 13. P. 15121. doi 10.1364/OE.21.015121

[15] Happer W., Tam A.C. // Phys. Rev. A. 1977. V. 16. N 5. P. 1877. doi 10.1103/PhysRevA.16.1877

[16] Happer $W / /$ Progr. Quant. Electron. 1970. V. 1. P. 51-103.

[17] Seltzer S.J. Developments in Alkali-Metal Atomic Magnetometry: Ph.D. Princeton University, 2008. 267 p.

[18] Вершовский А.К., Дмитриев С.П., Козлов Г.Г., Пазгалев А.С., Петренко М.В. // ЖТФ. 2020. Т. 90. № 8. C. 1243. doi 10.21883/JTF.2020.08.49533.438-19; Vershovskii A.K., Dmitriev S.P., Kozlov G.G., Pazgalev A.S., Petrenko M.V // Tech. Phys. 2020. V. 65. N 8. P. 1193. doi $10.1134 / \mathrm{S} 1063784220080204$ 